BMC

Research Notes

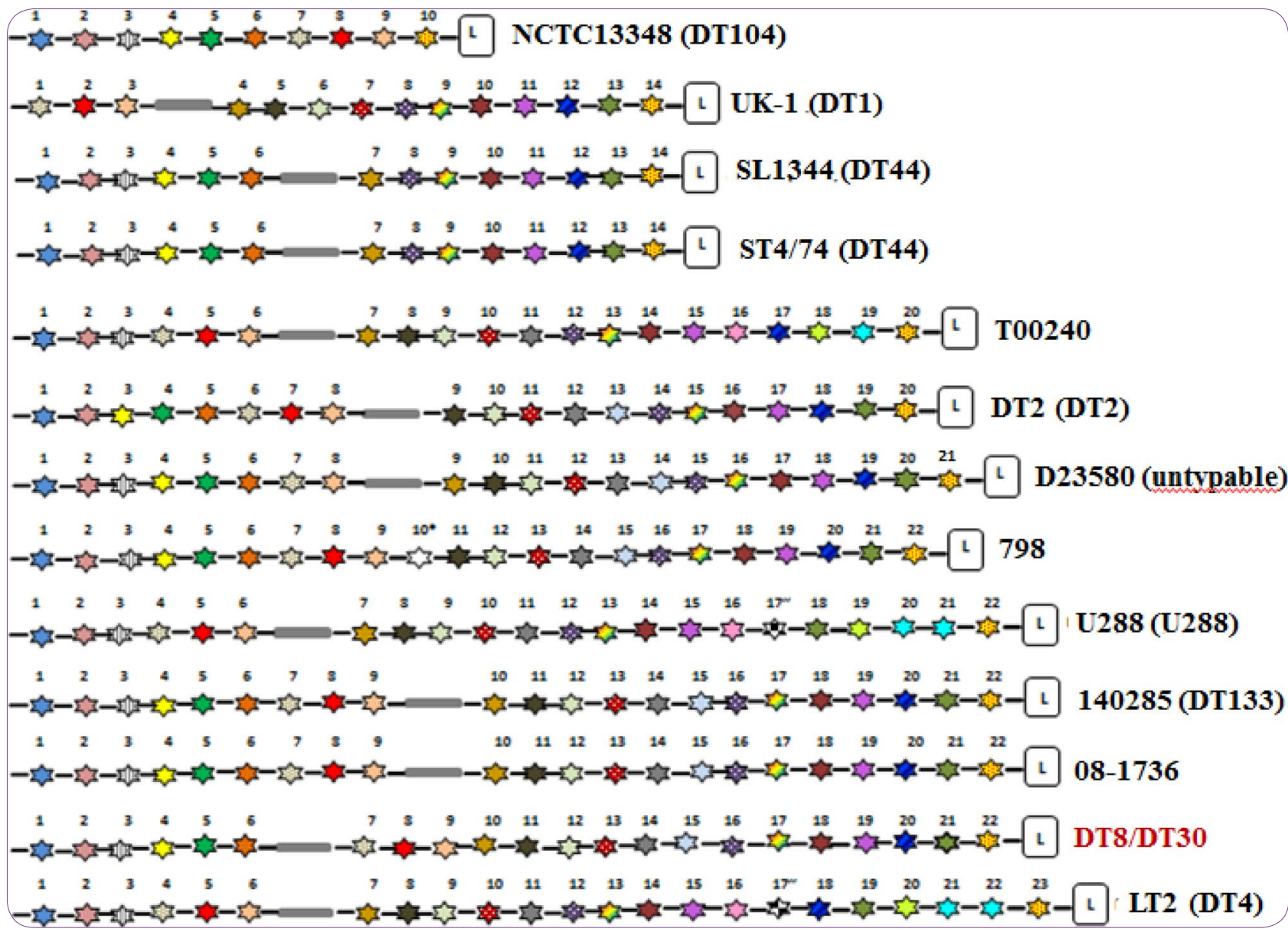

Whole genome sequencing provides possible explanations for the difference in phage susceptibility among two Salmonella Typhimurium phage types (DT8 and DT30) associated with a single foodborne outbreak Mohammed and Cormican 


\title{
Whole genome sequencing provides possible explanations for the difference in phage susceptibility among two Salmonella Typhimurium phage types (DT8 and DT30) associated with a single foodborne outbreak
}

\author{
Manal Mohammed ${ }^{*}$ and Martin Cormican
}

\begin{abstract}
Background: Phage typing has been used for decades as a rapid, low cost approach for the epidemiological surveillance of Salmonella enterica subsp. enterica serovar Typhimurium. Although molecular methods are replacing phage typing the system is still in use and provides a valuable model for study of phage-host interaction. Phage typing depends on the pattern of bacterial resistance or sensitivity to a panel of specific bacteriophages. In the phage typing scheme, S. Typhimurium definitive phage types (DT) 8 and 30 differ greatly in their susceptibility to the 30 typing phages of S. Typhimurium; DT8 is susceptible to 11 phages whereas DT30 is resistant to all typing phages except one phage although both DT8 and DT30 were reported to be associated with a single foodborne salmonellosis outbreak in Ireland between 2009 and 2011. We wished to study the genomic correlates of the DT8 and DT30 difference in phage susceptibility using the whole genome sequence (WGS) of S. Typhimurium DT8 and DT30 representatives.

Results: Comparative genome analysis revealed that both S. Typhimurium DT8 and DT30 are lysogenic for three prophages including two S. Typhimurium associated prophages (Gifsy-2 and ST64B) and one S. Enteritidis associated prophage (Enteritidis lysogenic phage S) which has not been detected previously in S. Typhimurium. Furthermore, DT8 and DT30 contain identical clustered regularly interspaced short palindromic repeats (CRISPRs). Interestingly, S. Typhimurium DT8 harbours an accessory genome represented by a virulence plasmid that is highly related to the PSLT plasmid of S. Typhimurium strain LT2 (phage typed as DT4) and codes a unique methyltransferase (MTase); M.EcoGIX related MTase. This plasmid is not detected in DT30. On the other hand, DT30 carries a unique genomic island similar to the integrative and conjugative element (ICE) of Enterotoxigenic Escherichia coli (ETEC) and encodes type IV secretion pathway system (T4SS) and several hypothetical proteins. This genomic island is not detected in DT8.

Conclusions: We suggest that differences in phage susceptibility between DT8 and DT30 may be related to acquisition of ICE in DT30 and loss of pSLT like plasmid that might be associated with DT30 resistance to almost all phages used in the typing scheme. Additional studies are required to determine the significance of the differences among DT8 and DT30 in relation to the difference in phage susceptibility. This study represents an initial step toward understanding the molecular basis of this host-phage relationship.
\end{abstract}

Keywords: Salmonella Typhimurium, Phage typing, DT8 and DT30, Comparative genomics

*Correspondence: drmanal20@hotmail.com

School of Medicine, National University of Ireland Galway, Galway, Ireland 


\section{Background}

Salmonellosis is an infectious disease affecting humans and almost all known animals. It is caused by members of the genus Salmonella. Salmonella enterica subsp. enterica serovar Typhimurium is a leading cause of foodborne illness worldwide. Routine epidemiological surveillance of $S$. Typhimurium infections has been performed for decades using $S$. Typhimurium phage typing scheme [1]. Phage typing depends on the pattern of bacterial resistance or sensitivity to a panel of $S$. Typhimurium specific bacteriophages. More than 300 definitive phage types (DTs) are recognised [2]. However, the evolutionary relationship among different phage types is not well described. Bacteria may have several antiviral mechanisms associated with resistance to infection by bacteriophages. These include masking the surface receptors by capsule or other surface components to block phage adsorption to the host cell [3]. Other systems within the bacteria are also involved including restriction modification (RM), clusterd regulatory interspaced short palindromic repeats (CRISPR) loci coupled to CRISPR associated sequence (CAS) proteins and superinfection exclusion (Sie) systems $[4,5]$.

Phage typing has proved to be very useful in investigating S. Typhimurium foodborne outbreaks. Two large $S$. Typhimiurium DT8 outbreaks associated with consumption of duck eggs were reported in Europe. In 2010 an outbreak of $S$. Typhimurium DT8 was reported in England and Northern Ireland [6]. Another outbreak of $S$. Typhimurium DT8 was reported in Ireland over a 19-month period between August 2009 and February 2011 [7]. The outbreak was also linked to the consumption of duck eggs where $S$. Typhimurium DT8 and DT30 were isolated. DT8 was more predominant during the outbreak and was isolated from humans and duck eggs however, DT30 were isolated from ducks during the outbreak (but non from humans). S. Typhimurium DT8 and DT30 are considered closely related but differ greatly in phage susceptibility. S. Typhimurium DT8 is susceptible to typing phage 8 and to varying degrees to 10 of 30 whereas DT30 is susceptible only to typing phage 8 as illustrated in Table 1. Here, we report genomic differences between DT8 and DT30 that may be relevant to this difference in phage susceptibility.

\section{Methods}

\section{Isolates selection and genomic DNA preparation}

Four representative isolates of $S$. Typhimurium DT8 and DT30 being used as control positive in Anderson typing scheme of $S$. Typhimurium were selected. Isolates included one DT30 (MS57) and three DT8 (PB225, PB469 and PB880) strains. Bacterial isolates were cultured on nutrient agar media and incubated overnight at $37^{\circ} \mathrm{C}$. Bacterial colonies were removed from the culture plate with an inoculation loop and genomic DNA was extracted using QIAamp ${ }^{\circledR}$ DNA Mini kit (Qiagen) according to manufacturer's instructions. DNA quality and quantity were checked by gel electrophoresis and Qubit $^{\circledR}$ quantification platform (Invitrogen) respectively. $20 \mu \mathrm{l}$ of DNA $(20-50 \mathrm{ng} / \mu \mathrm{l})$ from each isolate was submitted for Illumina sequencing.

\section{Genomic library preparation and sequencing}

Whole genome sequencing (WGS) was performed using an Illumina MiSeq on 250 bp paired-end (PE) libraries. The raw paired fastq sequence data were submitted to European Nucleotide Archive (ENA) http://www.ebi. ac.uk/ena/data/view/PRJEB8262.

Accession numbers are available in Table 2. We also included the whole genome of a clinical isolate of $S$. Typhimurium DT8 (ERS007592) that was isolated from human stool in 2009, UK [37] and the raw paired fastq sequence files for this isolate were downloaded from ENA http://www.ebi.ac.uk/ena/data/view/ ERR024405\&display $=\mathrm{html}$.

\section{Sequence data quality control}

The quality of PE Illumina sequence data for each isolate was evaluated using the FastQC toolkit (http://www.bioinformatics.babraham.ac.uk/projects/fastqc/). Adapter sequences were trimmed and low quality reads were removed using ea-utils package (https://code.google. com/p/ea-utils/).

\section{Read mapping and de novo sequence assembly}

Sequence reads from each isolate were mapped against the reference genome of $S$. Typhimurium strain LT2 (that belongs to phage type 4; DT4) along with its associated plasmid (pSLT) using Burrows Wheeler Aligner (BWA) [38]. Genomic variants including single nucleotide polymorphisms (SNPs) and insertions and deletions (indels) were identified using samtools mpileup [39] and filtered with a minimum mapping quality of 60 (i.e. 1 in 1,000,000 chance of a miss-called variant) are only accepted. Genuine SNPs were present in both forward and reverse direction and supported by $\geq 70 \%$ of the reads.

The impact of variants were evaluated using SnpEff program (http://snpeff.sourceforge.net/index.html). SNPs were compared to the reference genome of $S$. Typhimurium strain LT2 and a maximum likelihood (ML) phylogeny of the isolates was constructed using MEGA6 software [40]. Selection of the best-fit model for nucleotide substitution was carried out by jModelTest [41].

Reads were de novo assembled using Velvet [42]. The parameters ' $k$-mer length, expected coverage, coverage cut-off and insert length' were optimized to obtain the 


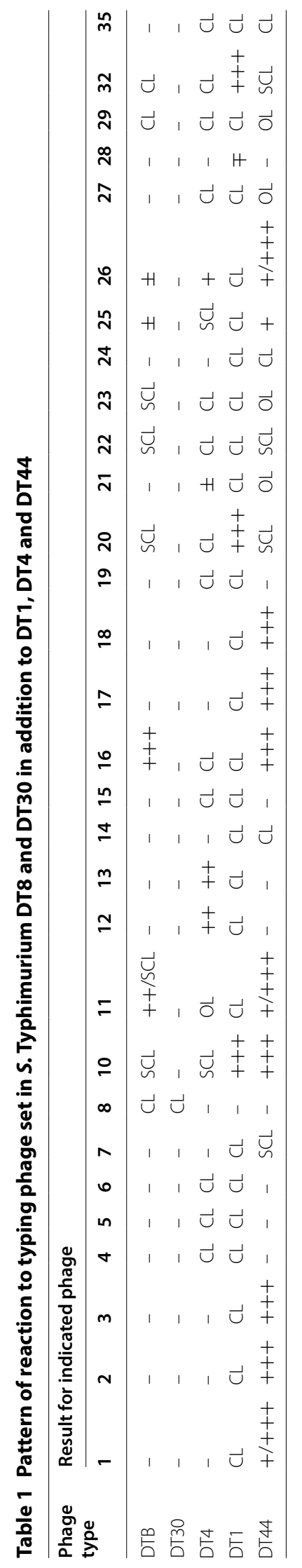


Table 2 List of the S. Typhimurium isolates used in this study

\begin{tabular}{llll}
\hline Isolate & $\begin{array}{l}\text { Phage } \\
\text { type (DT) }\end{array}$ & $\begin{array}{l}\text { Accession } \\
\text { number }\end{array}$ & References \\
\hline S. Typhimurium MS57 & DT30 & ERS640854 & This study \\
S. Typhimurium PB225 & DT8 & ERS640855 & This study \\
S. Typhimurium PB469 & DT8 & ERS640856 & This study \\
S. Typhimurium PB880 & DT8 & ERS640857 & This study \\
S. Typhimurium DT8 & DT8 & ERS007592 & {$[38]$} \\
S. Typhimurium LT2 & DT4 & AE006468 & {$[18]$} \\
S. Typhimurium UK-1 & DT1 & CP002614 & {$[19]$} \\
S. Typhimurium SL1344 & DT44 & FQ312003 & {$[20]$} \\
\hline
\end{tabular}

highest N50 value and the best possible assembly. Generated multi-contig draft genomes for each isolate were analysed and screened for genomic regions and structures that might be associated with phage susceptibility. Genome annotation was performed with the help of Rapid Annotation using Subsystem Technology (RAST) system [43].

\section{Results}

\section{Identification of SNPs and Indels}

Mapping short Illumina PE reads of the DT30 (MS57) isolate to the reference $S$. Typhimurium strain LT2 (phage typed as DT4) revealed 2538 SNPs and 96 indels within the bacterial chromosome. While the four DT8 isolates (PB225, PB469, PB880 and ERS007592) showed 2407, 2431, 2420 and 2096 SNPs respectively and 89, 97, 93 and 101 indels respectively. SNPs were randomly distributed around the bacterial chromosome. Variant call files (VCF) including SNPs and their effect for DT8 and DT30 chromosomes are provided in Additional file 1: Table S1. The majority of the SNPs were silent ranging from $63.2 \%$ in DT8 to $64.5 \%$ in DT30. Nonsense mutations varied from $0.5 \%$ in DT30 to $0.7 \%$ in DT8. The missense mutations ranged from $35 \%$ in DT30 to $36.04 \%$ in DT8. Nonsynonymous mutations are involved within the genes of the integrated prophages of the reference $S$. Typhimurium strain LT2 (DT4). Detailed gene-by-gene information is provided in Additional file 2: Table S2.

Visualization of the mapped reads and variants for each isolate using Integrative Genomics Viewer (IGV) [8] confirmed that the majority of the SNPs are located within the integrated prophages of the reference $S$. Typhimurium strain LT2. All variants within the prophages were therefore excluded to obtain 885 SNPs within DT30 (MS57) and 953, 939, 847 and 877 SNPs within DT8 isolates PB225, PB469, PB880 and ERS007592 respectively. SNPs within the chromosome (excluding prophages) of DT8 and DT30 isolates are provided in Additional file 3: Table S3. Although there are 716 SNPs common among both DT8 and DT30 isolates DT30 has 33 unique SNPs that do not occur in any of the DT8 strains. ML tree showed close relation among DT30 and DT8 strains with no significant divergence among them as illustrated in Fig. 1.

\section{De novo assembly}

De novo assembly of the 250 bp PE Illumina reads of the S. Typhimurium DT8 (PB225) isolate yielded an N50 scaffold size of $275,446 \mathrm{bp}$; largest scaffold $=660,336$ bp with median coverage depth $34 \times$, N50 of 83,098; largest scaffold $=263,713$ bp for DT8 (PB469) strain with median coverage depth $15.9 \times$, N50

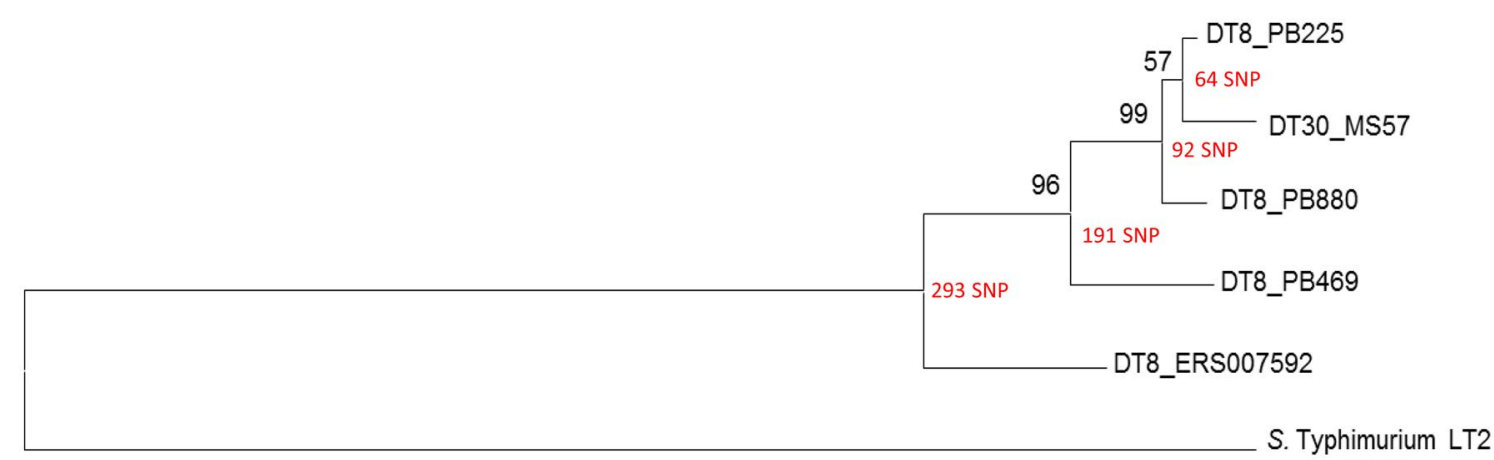

(DT4)

\section{2}

Fig. 1 Maximum-likelihood phylogenetic tree of S. Typhimurium strains. The tree is based on SNPs determined from the whole genome sequence (excluding prophage regions and phage remnant). Tree was inferred by using a general time-reversible (GTR) model with a gamma distribution. Bootstrap support values, given as a percentage of 1000 replicates, are shown on the branches. SNPs supporting each branch are displayed in red 
of $184,411 \mathrm{bp}$; largest scaffold $=286,128$ bp for DT8 (PB880) strain with median coverage depth $34 \times$, N50 of $377,002 \mathrm{bp}$; largest scaffold $=600,548 \mathrm{bp}$ for the DT8 (ERS007592) strain with median coverage depth $27.5 \times$ and N50 of 132,774 bp; largest scaffold $=568,574 \mathrm{bp}$ for the DT30 (MS57) variant with median coverage depth $29 \times$.

\section{Prophages in S. Typhimurium DT8 and DT30}

The draft genome of both $S$. Typhimurium DT8 and DT30 harbours three prophages as confirmed by PHAST [9] including phage Gifsy-2 (Figs. 2, 3), phage ST64B
(Fig. 3) and phage RE-2010 (Fig. 4) as well as a phage remnant (Figs. 2, 3). RE-2010 is a S. Enteritidis associated prophage belongs to Enteritidis lysogenic phage $S$ (ELPhiS) which has not been detected previously in any of the $S$. Typhimurium strains.

Mapping short Illumina PE reads of DT8 and DT30 to the reference genomes of phages Gifsy-2 (GenBank accession NC_010393), ST64B (GenBank accession AY055382) and RE-2010 (GenBank accession HM770079) revealed that the sequence of the prophage RE-2010 in both DT8 and DT30 is conserved. The prophage ST64B of DT8 has one coding SNP (at position 18582) located in $s b 25$ gene

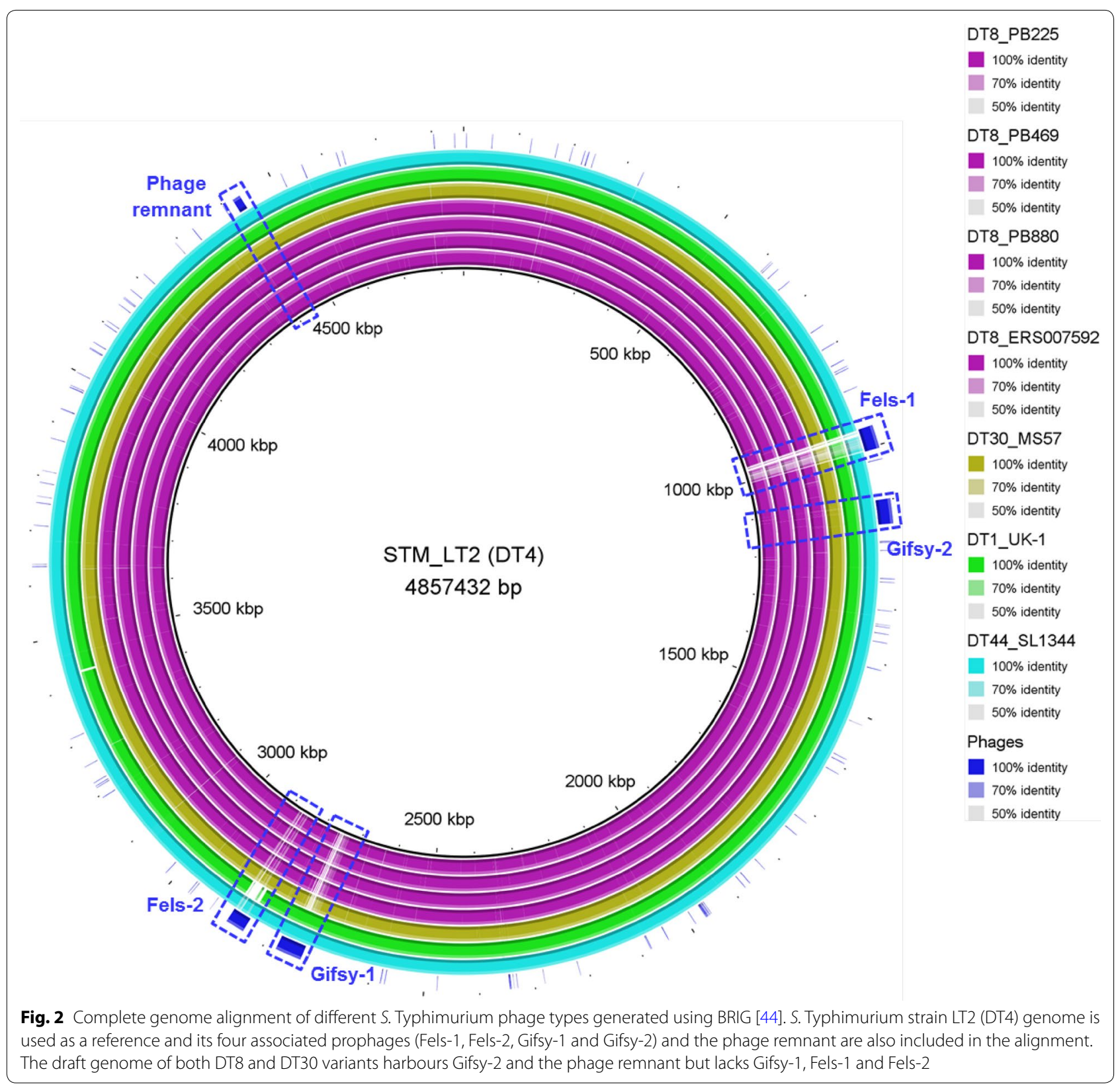






coding probable tail fibre protein that does not occur in the ST64B prophage of DT30 however, this SNP was synonymous and did not change the protein sequence. Prophage Gifsy-2 of DT8 has one synonymous SNP (at position 11178) coding bacteriophage damage-inducible protein DinI. Interestingly, prophage antitermination protein (locus_tag: STM1022) of phage Gifsy-2 has two SNPs including one synonymous SNP in DT8 in addition to one non-synonymous SNP in DT30 that changed the amino acid tryptophan (Trp, W) in DT8 to cysteine (Cys, C) in DT30.

\section{RMS in S. Typhimurium DT8 and DT30}

Salmonella Typhimurium DT8 and DT30 draft genomes contain the four types of restriction-modification systems (RMS); I, II, III and IV as illustrated in Table 3. However, DT8 carries an extra methyltransfearse (MTase) belonging to type II RMS that is closely related ( $84.25 \%$ identity) to M.EcoGIX MTase. Analysis of the RMS within other strains of $S$. Typhimurium belonging to different phage types including DT1 (strain UK-1), DT4 (strain LT2) and DT44 (strain SL1344) revealed that the closely related M.EcoGIX MTase is carried on the plasmids of 


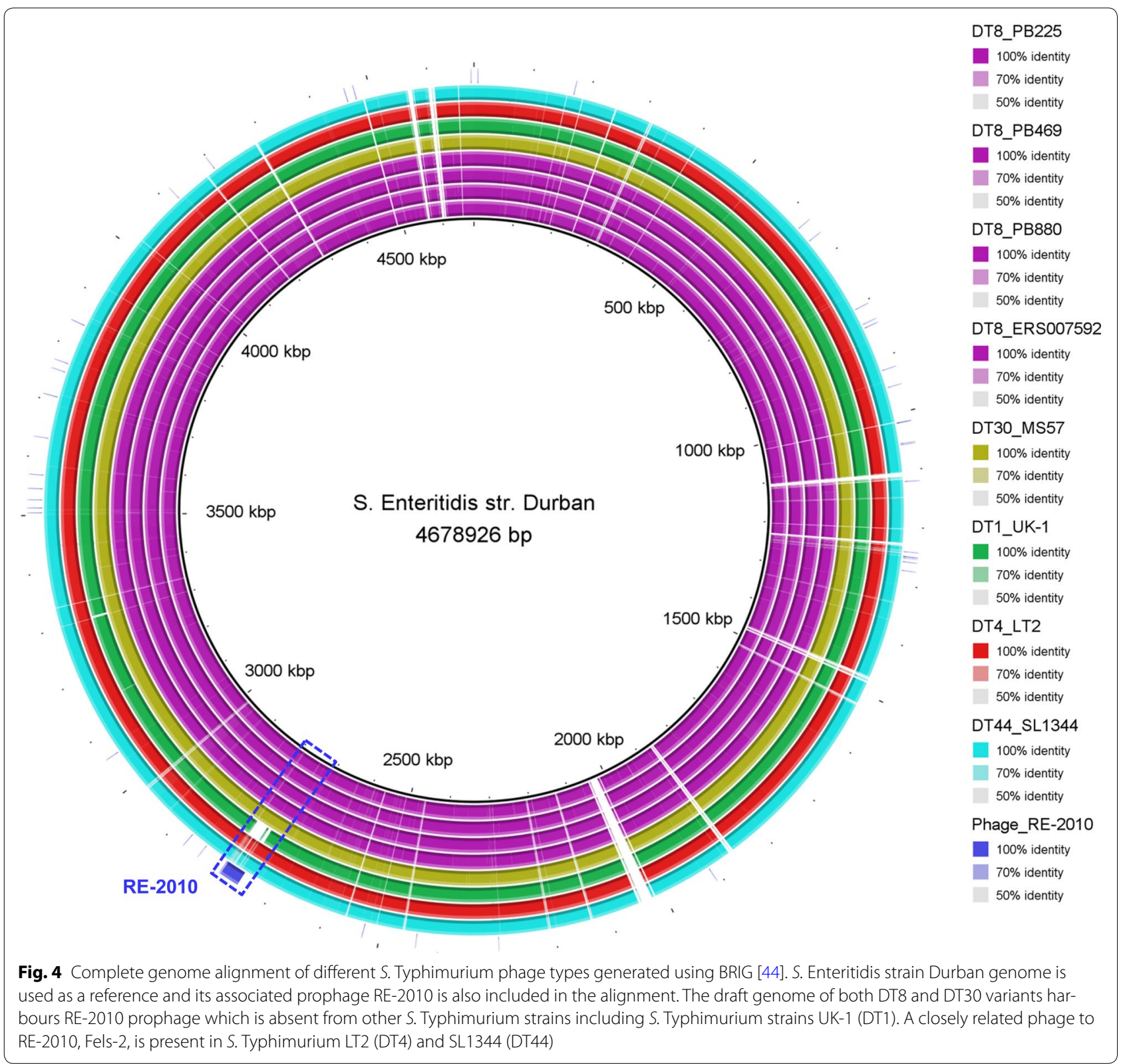

DT1, DT4 and DT44, M.SenTFII MTase (type I RMS) is unique to DT4 while absent from other phage types and M.Sen158III MTase (type II RMS) is unique to DT8 and DT30 while absent from DT1, DT4 and DT44.

\section{CRISPRs in S. Typhimurium DT8 and DT30}

Two CRISPR loci, CRISPR-1 and CRISPR-2, were detected within both $S$. Typhimurium DT8 and DT30 using CRISPRFinder [10]. The two variants, DT8 and DT30, contain highly similar palindromic repeats to other $S$. Typhimurium strains however their spacers are identical. DT8 and DT30 have the smallest number of spacers compared to the other $S$. Typhimurium strains as showed in Table 4 suggesting exposure to fewer phages. Analysis of CRISPR-1 locus among different $S$. Typhimurium phage types showed the variation in the number and pattern of spacers as illustrated in Fig. 5. However, some spacers are located at the same position within $S$. Typhimurium strains which is consistent with the hypothesis of common ancestor.

\section{Novel mobile genetic elements in S. Typhimurium DT8 and DT30}

The four representative $S$. Typhimurium DT8 draft genomes harbour a plasmid $(\sim 93 \mathrm{~kb})$ that is genetically 
Table 3 List of the four types of RMS present within different S. Typhimurium phage types as confirmed by REBASE [44]

\begin{tabular}{|c|c|c|c|c|c|c|c|}
\hline RMS genes & Function & Recognition sequence & DT1 & DT4 & DT44 & DT8 & DT30 \\
\hline \multicolumn{8}{|c|}{ Restriction modification system } \\
\hline \multicolumn{8}{|l|}{ Type I RMS } \\
\hline EcoKI & Restriction enzyme & AACNNNNNNGTGC & + & + & + & + & + \\
\hline M.Sen 1736III & Methyltransferase & GAGNNNNNNRTAYG & + & - & + & + & + \\
\hline S.Sen3181 & Specificity subunit & & + & + & + & + & + \\
\hline M.SenTFII & Methyltransferase & GAGNNNNNNRTAYG & - & + & - & - & - \\
\hline \multicolumn{8}{|l|}{ Type II RMS } \\
\hline M.Sen1736V & Methyltransferase & GATC & + & - & + & + & + \\
\hline M.Sen158IV & Methyltransferase & BATGCATV & + & + & + & + & + \\
\hline M.Sen 158/II & Methyltransferase & GATC & - & - & - & + & + \\
\hline M.SenAboDcm & Methyltransferase & CCWGG & + & + & + & + & + \\
\hline $\operatorname{Sen} 173611$ & Restriction enzyme/methyltransferase & GATCAG & + & + & + & + & + \\
\hline M.EcoGIX & Methyltransferase & SAY & + & + & + & + & - \\
\hline \multicolumn{8}{|l|}{ Type III RMS } \\
\hline $\operatorname{Sen} A Z I I$ & Restriction enzyme & & + & + & + & + & + \\
\hline M.Sen 17361 & Methyltransferase & CAGAG & + & + & + & + & + \\
\hline \multicolumn{8}{|l|}{ Type IV RMS } \\
\hline StyLT2Mrr & Methyl-directed restriction enzyme & & + & + & + & + & + \\
\hline
\end{tabular}

Table 4 Distribution of the CRISPR loci detected in 13 different strains of S. Typhimurium

\begin{tabular}{|c|c|c|c|c|c|}
\hline Strain & $\begin{array}{l}\text { Phage } \\
\text { type }\end{array}$ & $\begin{array}{l}\text { Accession } \\
\text { number }\end{array}$ & $\begin{array}{l}\text { Total no. of spacers } \\
\text { in CRISPR loci }\end{array}$ & $\begin{array}{l}\text { Spacers no. In CRISPR-1 } \\
\text { (CRISFK-1 coordinates) }\end{array}$ & $\begin{array}{l}\text { Spacers no. In CRISPR-2 } \\
\text { (CRISPR-2 coordinates) }\end{array}$ \\
\hline UK-1 & DTI & CP002614 & 37 & $14(3045088-3046075)$ & $23(3062207-3063639)$ \\
\hline DT2 & DT2 & HG326213 & 46 & $20(3035608-3036960)$ & 26 (3053092-3054707) \\
\hline LT2 & DT4 & AE006468 & 55 & $23(3076611-3078147)$ & $32(3094279-3096260)$ \\
\hline SL1344 & DT44 & FQ312003 & 37 & 14 (3099172-3100159) & $23(3116291-3117723)$ \\
\hline ST4/74 & DT44 & CP002487 & 37 & 14 (3099172-3100159) & $23(3116291-3117723)$ \\
\hline NCTC13348 & DT104 & HF937208 & 36 & $10(3111855-3112493)$ & $26(3128625-3130239)$ \\
\hline $14028 \mathrm{~S}$ & DT133 & CP001363 & 48 & $22(3096848-3098323)$ & $26(3114455-3116070)$ \\
\hline $08-1736$ & - & CP006602 & 48 & 22 (4332390-4333865) & 26 (4349997-4351612) \\
\hline U288 & U288 & CP003836 & 54 & $22(3074262-3075737)$ & 32 (3091869-3093850) \\
\hline 798 & - & CP003386 & 44 & 22 (3097927-3099324) & $22(3115456-3116827)$ \\
\hline D23580 & Untypable & FN424405 & 39 & 21 (3069598-3071012) & IS (3087144-3088271) \\
\hline T000240 & DT12 & AP011957 & 52 & $20(3100041-3101393)$ & $32(3117525-3119506)$ \\
\hline PB225 & DT8 & ERS640E55 & 31 & 22 & 9 \\
\hline MS57 & DT30 & ERS640854 & 31 & 22 & 9 \\
\hline
\end{tabular}

Each strain (phage type) contains a variable number of spacers and both DT8 and DT30 have the lowest number of spacers

SL1344 and ST4/74 strains contain identical spacers and both belong to the same phage type (DT44)

UK-1 (DT1) has the same number of spacers as SL1344 (DT44) and ST4/74 (DT44), however they belong to a different phage type as DT1 has 6 unique spacers that do not occur in DT44 variants

(-) indicates that phage type is not known

related to the virulence pSLT plasmid of $S$. Typhimurium strain LT2. Two plasmid replicons were identified in DT8 draft genomes using PlasmidFinder [11]. DT8 specific plasmid differs from the pSLT plasmid in 13-17 SNPs and 1-4 indels (total variants are illustrated in Additional file 4: Table S4). DT8 specific plasmid has four coding nonsynonyms SNPs in locus tags; pSLT014, pSLT057 and
pSLT079 coding putative outer membrane protein, putative cytoplasmic protein, conjugal transfer pilus assembly protein (traE) respectively. Although DT30 lacks the pSLT related plasmid it carries a distinctive chromosomal mobile element absent from DT8 as illustrated in Fig. 6 . This element $(\sim 60 \mathrm{~kb})$ identified by BLAST [12] as an integrative and conjugative element (ICE) that 


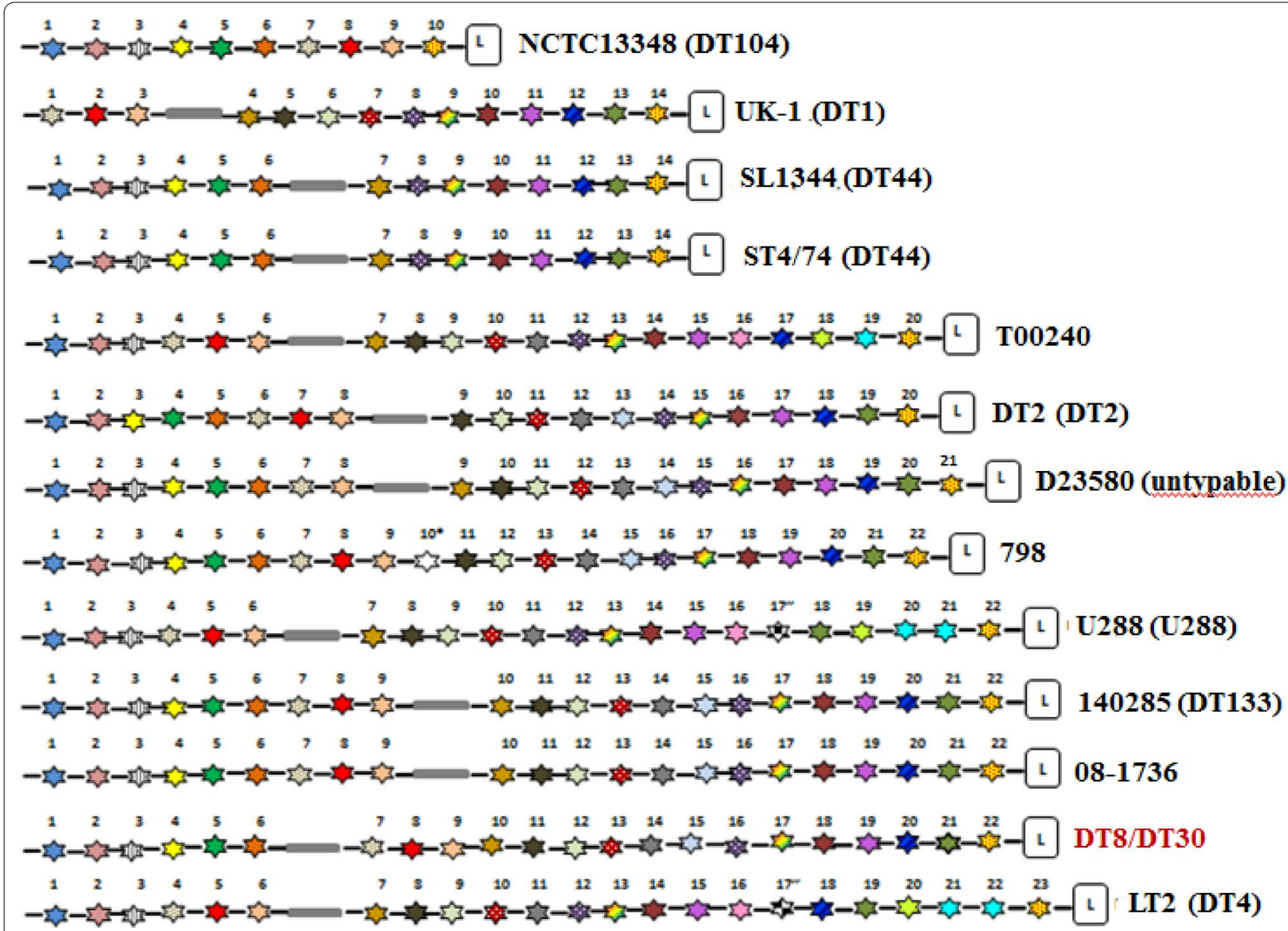

Fig. 5 Overview of CRISPR-1 locus in different strains of S. Typhimurium. Leader is shown as (L). Repeats are similar and shown as black lines. Spacers are shown as coloured stars. Spacers with identical sequence are shown in the same colour. White stars represent strain specific spacers. DT8 and DT30 have identical spacers. Strain 798 has a unique spacer (10*). Strains LT2 (DT4) and U288 (U288) share a unique spacer (17")

is genetically related to the ICE ( $75 \%$ identity) of the Enterotoxigenic Escherichia coli (ETEC) strain UMNK88 (GenBank accession CP002729) [13]. This novel mobile element encodes a type IV secretion pathway system (T4SS) as confirmed by Pfam [14] and several hypothetical proteins of unknown function (annotation of the ICE is provided in Additional file 5: Table S5).Analysis of the complete genome of other $S$. Typhimurium strains including UK-1 (DT1) and SL1344 (DT44) revealed the absence of ICE but the presence of a plasmid closely related to the pSLT plasmid of $S$. Typhimurium strain LT2 that has been also detected in S. Typhimurium DT8 but missing from DT30.

\section{Discussion}

Phage typing has been used for more than four decades as a rapid, low cost approach in epidemiological characterization of $S$. Typhimurium however, the underlying molecular basis of phage typing is not well described.
Although phage typing has certain limitations [15] it is still in use and represents a convenient model for studying phage-host interactions. In the $S$. Typhimurium phage typing scheme, bacterial strains are classified into more than 300 DTs based on the pattern of their sensitivity or resistance to a set of the typing phages. Interestingly, DT8 and DT30 have distinct pattern in phage susceptibility (DT30 is resistant to all typing phages except phage 8 whereas DT8 is susceptible to eleven phages) although both phage types were reported to be associated with a single foodborne salmonellosis outbreak in Ireland between August 2009 and February 2011. Here we apply WGS technology to investigate the genomic correlates of the striking difference in phage susceptibility among $S$. Typhimurium phage types, DT8 and DT30.

Bacterial antiphage systems include blockage of phage DNA entry through a phenomenon known as 'Sie' where existing prophage within bacterial genome prevents 


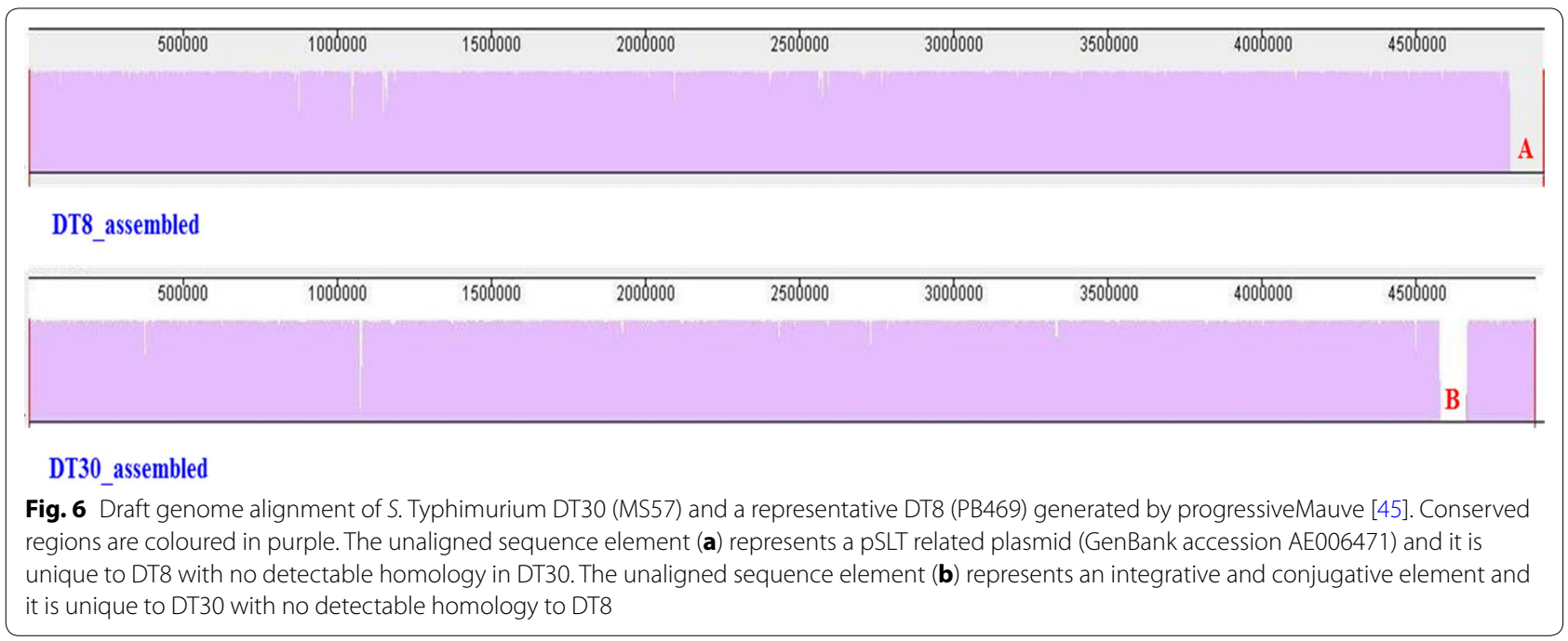

infection by the same or closely related viruses [16]. Integrated prophage can block phage DNA penetration into bacterial cell as it codes blocking proteins that are localized at the membrane/cell wall level of the host cell. A strong relation between the integrated prophages and the phage type was observed [17]. For example, $S$. Typhimurium strain LT2 contains 4 prophages; (Fels-1, Fels-2, Gifsy-1 and Gifsy-2) [18], S. Typhimurium strain UK-1 contains 3 prophages, two that are homologous to those in LT2 (Gifsy-1, Gifsy-2) in addition to ST64B prophage [19] and S. Typhimurium strain SL1344 contains 4 prophages; 3 homologs of those in LT2 (Gifsy-1, Gifsy2, Fels-2) and ST64B [20] and each of these strains has a distinct typing pattern as LT2, UK-1 and SL1344 strains belong to DT4, DT1 and DT44 respectively. However, in this study we found that both $S$. Typhimurium DT8 and DT30 contain the same 3 prophages. Two prophages are associated with $S$. Typhimurium including Gifsy-2 (also present in DT4) and ST64B (detected also in DT1). In addition, RE-2010, an $S$. Enteritidis associated prophage, that is closely related to Fels-2 phage [21]. This study is the first to report the presence of ELPhiS within $S$. Typhimurium strains.

Interestingly, prophage Gifsy-2 of DT30 has a unique non-synonymous SNP in the prophage antitermination protein that changed the amino acid tryptophan (Trp, W) in DT8 to cysteine (Cys, C) in DT30. Replacement of tryptophan by cysteine could negatively affect the function of antitermination protein since tryptophan is a unique amino acid in terms of chemistry and size.

CRISPR-Cas system has also been described as one of the prokaryotic antiviral defence systems [22]. It is considered as the adaptive bacterial immune system that provides acquired immunity against foreign DNA through targeting invading DNA in a sequence-specific manner. CRISPRs are acquired from invading phages and/or plasmids and incorporated within bacterial genome. These palindromic repeats are interspaced by spacers (interspaced regions between palindromic repeats) which are useful in providing information on the past exposure of the bacteria to foreign DNA including phages and/or plasmids. The immunization process is based on neutralizing foreign DNA through a mechanism similar to RNA interference (RNAi) [23]. It is considered that a strain with more spacers is consistent with exposure to more phages and DNA invasion along the lineage of that strain. However, a recent study suggested that Salmonella CRISPR-Cas systems are not immunogenic anymore [24].

CRISPRs were found to be variable among different $S$.Typhimurium phage types [19] and a strong correlation between CRISPR and phage type was reported and it has been suggested that CRISPR typing might be a powerful laboratory method for surveillance of Salmonella infections [25]. However, the presence of identical palindromic repeats interspaced with identical spacers among $S$. Typhimurium DT8 and DT30 analyzed in this study revealing the limitations of CRISPR typing for Salmonella surveillance.

It has been reported that genetic diversity among different phage types of $S$. Typhimurium is mainly due to accumulation of SNPs [26] which can result in gene inactivation. Interestingly, SNP typing of $S$. Typhimurium has been recommended to have the potential to replace the phage typing scheme [27]. However, our phylogenetic analysis based on SNPs determined from WGS of DT8 and DT30 did not reveal significant genetic divergence among the two phage types and SNPs were randomly distributed around bacterial chromosome. In fact, 
the historical control DT8 isolates (PB225, PB469 and PB880) and DT30 isolate (MS57) are more closely related to each other than to the recently isolated clinical DT8 isolate (ERS007592) suggesting that DT30 might have arisen from DT8.

RMS allows bacteria to recognize and destroy viral DNA by restriction endonucleases (REases) that have the ability to cut foreign DNA at certain sequence (restriction sites) while bacterial DNA is protected by the aid of DNA MTase that modifies restriction sites within the bacterial genome $[28,29]$. S. Typhimurium DT8 and DT30 contain similar RMS however DT8 has an extra MTase that is very similar to M.EcoGI ( $84.25 \%$ identity). M.EcoGIX MTase belongs to type II RMS and is a very unusual kind of MTase as it methylates only one of the double strands of DNA and it can modify adenines in a wide variety of sequence contexts [30]. Recently, it has been shown that M.EcoGIX MTase plays an important role in protecting the harbouring plasmid from digestion by host-encoded REases and subsequently expanding the plasmid host range [31]. We speculate that M.EcoGIX related MTase may play a similar role in protection of phage DNA thus allowing phage to multiply within bacterial cells. Several Bacillus subtilis phages incorporate modified bases into their genome [32,33] however it is not known if this is true for the $S$. Typhimurium typing phage. It is also possible that M.EcoGIX related MTase may be required for transcription of certain phage genes ensuring phage replication as reported earlier [34]. We note however that the methyl-directed REase (StyLT2Mrr) within the chromosome of both DT8 and DT30 appears to have the ability to cleave modified bases.

M.EcoGIX related MTase is carried on the plasmid of $S$. Typhimurium DT8 strains. The plasmid is genetically related to the pSLT plasmid of $S$. Typhimurium strain LT2 (DT4). Closely related plasmids to pSLT including pSTUK-100 and pSLT-SL1344 were also detected within S. Typhimurium strains UK-1 (DT4) and SL1344 (DT44) respectively. Acquisition of these closely related plasmds might be linked with the differences in phage susceptibility since an earlier study showed the change between phage types in $S$. Enteritidis was related to acquisition of a plasmid [35].

Salmonella Typhimurium DT30 does not carry any accessory genomes including PSLT related plasmid but it harbours a unique genetic mobile element, ICE, which is absent from the DT8 strains as well as other phage types including DT1, DT4 and D44. Sequence analysis of specific DT30 ICE did not reveal the presence of any REases or MTases that might be associated with bacterial resistance to almost all phages used in the typing scheme but ICE harbours T4SS and several orphan genes coding hypothetical proteins (proteins of unknown functions) that might play a role in bacterial reaction to bacteriophages. Few reports showed that ICE might harbour genes allowing bacteria to grow in the presence of harsh environment containing antibiotics, heavy metals as well as bacteriophages [36, 37]. However additional studies including prediction of the structure and function of ICE hypothetical proteins within DT30 are required in order to evaluate the role of ICE in bacterial resistance to phages.

The high similarity among the genome of DT8 and DT30 at the whole genome level does not explain the distinct differences in phage susceptibility among the two phage types. However, we suggest that the acquisition of novel genetic elements including the pSLT like plasmid in DT8 (absent from DT30) and/or the ICE in DT30 (absent from DT8) are significant in the distinct difference in phage susceptibility among the two phage types. Further experimental work is required to explore the hypothesis generated by this project which may have broader relevance in understanding phage-host interactions.

\section{Conclusions}

Salmonella Typhimurium phage typing scheme represents a good model for studying phage-host interactions. Comparative genomic analysis of the two $S$. Typhimurium DT8 and DT30 associated with a single foodborne outbreak represents an initial step toward understanding genetic basis of this $S$. Typhimurium phage-host interaction. The great difference in phage susceptibility between DT8 and DT30 and the identification of discrete points of differences in otherwise very similar strains makes this a useful model for further study of phage-host interaction. Additional studies are required to determine the significance of the differences among DT8 and DT30 in relation to the difference in phage susceptibility. Understanding the molecular basis for phage-host interactions may ultimately lead to engineering of lysogenic bacteriophage with specific spectra that may be used for treatment of resistant bacterial infections or for delivering antimicrobial agents to resistant bacteria.

\section{Additional files}

Additional file 1: Table S1. VCFs of DT8 and DT30 including SNPs and their effect.

Additional file 2: Table S2. Gene-by-gene information for DT8 and DT30 variants.

Additional file 3: Table S3. SNPs within the chromosome (excluding prophages) of DT8 and DT30 isolates.

Additional file 4: Table S4. Total variants among PSLT plasmid and the DT8 specific plasmid.

Additional file 5: Table S5. Annotation of the integrative and conjugative element of the DT30 isolate. 


\section{Abbreviations}

CRISPR: clustered regularly interspaced short palindromic repeats; DT: definitive phage type; ENA: European nucleotide archive; ETEC: enterotoxigenic Escherichia coli; ICE: integrative and conjugative element; IGV: integrative genomics viewer; Indels: insertions and deletions; MTase: methyltransferase; REase: restriction endonuclease; RMS: restriction-modification system; Sie: SUPERINFECTION exclusion; SNP: single nucleotide polymorphism; S. Typhimurium: Salmonella enterica subsp. enterica serovar Typhimurium; T4SS: Type IV secretion pathway system; VCF: variant call file; WGS: whole genome sequencing.

\section{Authors' contributions}

MM: designed the study, performed the laboratory work, carried out the bioinformatics analyses and interpretation of the data and drafted the manuscript. MC: critically revised the manuscript. Both authors read and approved the final manuscript.

\section{Acknowledgements}

Research funded from internal department budget, no external funding source.

\section{Competing interests}

The authors declare that they have no competing interests.

Received: 11 May 2015 Accepted: 10 November 2015 Published online: 27 November 2015

\section{References}

1. Anderson ES, Ward LR, Saxe MJ, de Sa JD. Bacteriophage-typing designations of Salmonella Typhimurium. J Hyg. 1977;78(2):297-300.

2. Rabsch W. Salmonella Typhimurium phage typing for pathogens. Methods Mol Biol. 2007:394:177-211.

3. Hyman P, Abedon ST. Bacteriophage host range and bacterial resistance. Adv Appl Microbiol. 2010;70:217-48.

4. Labrie SJ, Samson JE, Moineau S. Bacteriophage resistance mechanisms. Nat Rev Microbiol. 2010;8(5):317-27.

5. Dupuis ME, Villion M, Magadan AH, Moineau S. CRISPR-Cas and restriction-modification systems are compatible and increase phage resistance. Nat Commun. 2013:4:2087.

6. Noble DJ, Lane C, Little CL, Davies R, De Pinna E, Larkin L, Morgan D. Revival of an old problem: an increase in Salmonella enterica serovar Typhimurium definitive phage type 8 infections in 2010 in England and Northern Ireland linked to duck eggs. Epidemiol Infect. 2012;140(1):146-9.

7. Garvey P, McKeown P, Kelly P, Cormican M, Anderson W, Flack A, Barron S, De Lappe N, Buckley J, Cosgrove C, et al. Investigation and management of an outbreak of Salmonella Typhimurium DT8 associated with duck eggs, Ireland 2009-2011. Euro Surveill Bull Eur Sur Les maladies Transm Eur Commun Dis Bull. 2013;18(16):20454

8. Thorvaldsdottir H, Robinson JT, Mesirov JP. Integrative genomics viewer (IGV): high-performance genomics data visualization and exploration. Brief Bioinform. 2013;14(2):178-92.

9. Zhou Y, Liang Y, Lynch KH, Dennis JJ, Wishart DS. PHAST: a fast phage search tool. Nucleic Acids Res. 2011:39(Web Server issue):W347-52.

10. Grissa I, Vergnaud G, Pourcel C. CRISPRFinder: a web tool to identify clustered regularly interspaced short palindromic repeats. Nucleic Acids Res. 2007;35(Web Server issue):W52-7.

11. Carattoli A, Zankari E, Garcia-Fernandez A, Voldby Larsen M, Lund O, Villa L, Moller Aarestrup F, Hasman $\mathrm{H}$. In silico detection and typing of plasmids using plasmid finder and plasmid multilocus sequence typing. Antimicrob Agents Chemother. 2014;58(7):3895-903.

12. Altschul SF, Gish W, Miller W, Myers EW, Lipman DJ. Basic local alignment search tool. J Mol Biol. 1990;215(3):403-10.

13. Shepard SM, Danzeisen $J$, Isaacson RE, Seemann T, Achtman M, Johnson TJ. Genome sequences and phylogenetic analysis of K88and F18-positive porcine enterotoxigenic Escherichia coli. J Bacteriol. 2012;194(2):395-405.
14. Finn RD, Bateman A, Clements J, Coggill P, Eberhardt RY, Eddy SR, Heger A, Hetherington K, Holm L, Mistry J, et al. Pfam: the protein families database. Nucleic Acids Res. 2014;42(Database issue):D222-30.

15. Baggesen DL, Sorensen G, Nielsen EM, Wegener HC. Phage typing of Salmonella Typhimurium - is it still a useful tool for surveillance and outbreak investigation? Euro Surveill Bull Eur Sur Les Mal Transm Eur Commun Dis Bull. 2010;15(4):19471.

16. Roossinck MJ. The good viruses: viral mutualistic symbioses. Nat Rev Microbiol. 2011;9(2):99-108.

17. Hiley L, Fang NX, Micalizzi GR, Bates J. Distribution of Gifsy-3 and of variants of ST64B and Gifsy-1 prophages amongst Salmonella enterica Serovar Typhimurium isolates: evidence that combinations of prophages promote clonality. PLoS One. 2014;9(1):e86203.

18. McClelland M, Sanderson KE, Spieth J, Clifton SW, Latreille P, Courtney L, Porwollik S, Ali J, Dante M, Du F, et al. Complete genome sequence of Salmonella enterica serovar Typhimurium LT2. Nature. 2001;413(6858):852-6.

19. Luo Y, Kong Q, Yang J, Mitra A, Golden G, Wanda SY, Roland KL, Jensen RV, Ernst PB, Curtiss R 3rd. Comparative genome analysis of the high pathogenicity Salmonella Typhimurium strain UK-1. PLoS One. 2012;7(7):e40645

20. Kroger C, Dillon SC, Cameron AD, Papenfort K, Sivasankaran SK, Hokamp K, Chao Y, Sittka A, Hebrard M, Handler K, et al. The transcriptional landscape and small RNAs of Salmonella enterica serovar Typhimurium. Proc Natl Acad Sci USA. 2012;109(20):E1277-86.

21. Hanna LF, Matthews TD, Dinsdale EA, Hasty D, Edwards RA. Characterization of the ELPhiS prophage from Salmonella enterica serovar Enteritidis strain LK5. Appl Environ Microbiol. 2012;78(6):1785-93.

22. Barrangou R, Horvath P. CRISPR: new horizons in phage resistance and strain identification. Annu Rev Food Sci Technol. 2012;3:143-62.

23. Makarova KS, Grishin NV, Shabalina SA, WolfYI, Koonin EV. A putative RNAinterference-based immune system in prokaryotes: computational analysis of the predicted enzymatic machinery, functional analogies with eukaryotic RNAi, and hypothetical mechanisms of action. Biol Direct. 2006;1:7.

24. Shariat N, Timme RE, Pettengill JB, Barrangou R, Dudley EG. Characterization and evolution of Salmonella CRISPR-Cas systems. Microbiology. 2015;161(Pt 2):374-86.

25. Fabre L, Zhang J, Guigon G, Le Hello S, Guibert V, Accou-Demartin M, de Romans S, Lim C, Roux C, Passet V, et al. CRISPR typing and subtyping for improved laboratory surveillance of Salmonella infections. PLoS One. 2012;7(5):e36995.

26. Pang S, Octavia S, Feng L, Liu B, Reeves PR, Lan R, Wang L. Genomic diversity and adaptation of Salmonella enterica serovar Typhimurium from analysis of six genomes of different phage types. BMC Genom. 2013;14:718.

27. Pang S, Octavia S, Reeves PR, Wang Q, Gilbert GL, Sintchenko V, Lan R. Genetic relationships of phage types and single nucleotide polymorphism typing of Salmonella enterica Serovar Typhimurium. J Clin Microbiol. 2012;50(3):727-34

28. Roberts RJ, Belfort M, Bestor T, Bhagwat AS, Bickle TA, Bitinaite J, Blumenthal RM, Degtyarev S, Dryden DT, Dybvig K, et al. A nomenclature for restriction enzymes, DNA methyltransferases, homing endonucleases and their genes. Nucleic Acids Res. 2003;31(7):1805-12.

29. Wilson GG, Murray NE. Restriction and modification systems. Annu Rev Genet. 1991;25:585-627.

30. Fang G, Munera D, Friedman DI, Mandlik A, Chao MC, Banerjee O, Feng Z, Losic B, Mahajan MC, Jabado OJ, et al. Genome-wide mapping of methylated adenine residues in pathogenic Escherichia coli using singlemolecule real-time sequencing. Nat Biotechnol. 2012;30(12):1232-9.

31. Yamaichi Y, Chao MC, Sasabe J, Clark L, Davis BM, Yamamoto N, Mori H, Kurokawa K, Waldor MK. High-resolution genetic analysis of the requirements for horizontal transmission of the ESBL plasmid from Escherichia coli O104:H4. Nucleic Acids Res. 2015;43(1):348-60.

32. Kruger DH, Bickle TA. Bacteriophage survival: multiple mechanisms for avoiding the deoxyribonucleic acid restriction systems of their hosts. Microbiol Rev. 1983:47(3):345-60.

33. Warren RA. Modified bases in bacteriophage DNAs. Annu Rev Microbiol. 1980;34:137-58.

34. Hattman S. DNA methyltransferase-dependent transcription of the phage Mu mom gene. Proc Natl Acad Sci USA. 1982;79(18):5518-21.

35. Frost JA, Ward LR, Rowe B. Acquisition of a drug resistance plasmid converts Salmonella enteritidis phage type 4 to phage type 24 . Epidemiol Infect. 1989:103(2):243-8. 
36. Burrus $V$, Waldor MK. Shaping bacterial genomes with integrative and conjugative elements. Res Microbiol. 2004;155(5):376-86.

37. Burrus V, Pavlovic G, Decaris B, Guedon G. Conjugative transposons: the tip of the iceberg. Mol Microbiol. 2002;46(3):601-10.

38. Okoro CK, Kingsley RA, Connor TR, Harris SR, Parry CM, Al-Mashhadani MN, Kariuki S, Msefula CL, Gordon MA, de Pinna E, et al. Intracontinental spread of human invasive Salmonella Typhimurium pathovariants in subSaharan Africa. Nat Genet. 2012;44(11):1215-21.

39. Li H, Durbin R. Fast and accurate short read alignment with BurrowsWheeler transform. Bioinformatics. 2009;25(14):1754-60.

40. Li H, Handsaker B, Wysoker A, Fennell T, Ruan J, Homer N, Marth G, Abecasis G, Durbin R. The Sequence Alignment/Map format and SAMtools. Bioinformatics. 2009;25(16):2078-9.
41. Tamura K, Stecher G, Peterson D, Filipski A, Kumar S. MEGA6: molecular evolutionary genetics analysis version 6.0. Mol Biol Evol. 2013;30(12):2725-9.

42. Posada D. jModelTest: phylogenetic model averaging. Mol Biol Evol. 2008;25(7):1253-6.

43. Zerbino DR, Birney E. Velvet: algorithms for de novo short read assembly using de Bruijn graphs. Genome Res. 2008;18(5):821-9.

44. Aziz RK, Bartels D, Best AA, DeJongh M, Disz T, Edwards RA, Formsma K, Gerdes S, Glass EM, Kubal M, et al. The RAST server: rapid annotations using subsystems technology. BMC Genom. 2008;9:75.

45. Roberts RJ, Vincze T, Posfai J, Macelis D. REBASE - a database for DNA restriction and modification: enzymes, genes and genomes. Nucleic Acids Res. 2010;38(Database issue):D234-6.
Submit your next manuscript to BioMed Central and take full advantage of:

- Convenient online submission

- Thorough peer review

- No space constraints or color figure charges

- Immediate publication on acceptance

- Inclusion in PubMed, CAS, Scopus and Google Scholar

- Research which is freely available for redistribution

Submit your manuscript at

www.biomedcentral.com/submit

O) BioMed Central 